\title{
ORIGINAL
}

\section{EVALUACIÓN ECONÓMICA DEL IMPACTO DE LA PROHIBICIÓN DE PLOMO EN LAS GASOLINAS EN EL COCIENTE INTELECTUAL DE LOS NIÑOS DE 7 Y 8 AÑOS DE LA COMUNIDAD DE MADRID}

\author{
José Ma Ordóñez-Iriarte (1), José Jesús Guillén-Pérez (2), Andrés Bodas-Pinedo (3), Manuel \\ Ignacio Aparicio-Madre (4), María José Martínez-García (5), Montserrat González-Estecha (3) \\ y Juan Manuel Cabasés-Hita (6).
}

(1) Dirección General de Salud Pública. Consejería de Sanidad. Comunidad de Madrid.

(2) Departamento de Ciencias Sociosanitarias. U.D. Medicina Preventiva y Salud Pública. Universidad de Murcia.

(3) Hospital Clínico San Carlos. Consejería de Sanidad. Comunidad de Madrid.

(4) Dirección General de Coordinación de la Asistencia Sanitaria. Consejería de Sanidad. Comunidad de Madrid.

(5) Departamento de Ingeniería Química y Ambiental. Universidad Politécnica de Cartagena.

(6) Departamento de Economía. Universidad Pública de Navarra.

Los autores manifiestan que no existe ningún conflicto de interés.

\section{RESUMEN}

Fundamentos: La evaluación económica de las repercusiones sanitarias de las políticas públicas es difícil y escasa. El objetivo del presente estudio fue cuantificar monetariamente los beneficios de la reducción del plomo en la sangre de los niños de 7-8 años en la Comunidad de Madrid (España), derivada de la prohibición del plomo en las gasolinas.

Métodos: Se calculó la disminución de puntos de cociente intelectual (CI), utilizando dos estudios realizados en niños de 7 a 8 años según los valores de plomo en sangre que presentaban: media geométrica de $3,8 \mu \mathrm{g}$ $\mathrm{dL}$ en 1995 y $0,9 \mu \mathrm{g} / \mathrm{dL}$ en 2010 . La mejoría neta de CI se midió en términos de capacidad de producción ganada a lo largo de la vida laboral, siguiendo los métodos de Schwartz y Salkiver.

Resultados: La reducción de los niveles de plomo en sangre de estos niños evitó perder entre 135.391 y 144.153 puntos de CI. La valoración económica actual de estos puntos en términos de capacidad de producción ganada a lo largo de la vida laboral de estos sujetos se estimó en un rango entre 626,4 y 865,4 millones de euros (valor 2009).

Conclusiones: Las cifras de beneficios económicos encontradas derivadas de la medida de prohibición de uso de plomo en las gasolinas son importantes en términos sociales.

Palabras clave: Niño. Gasolina. Contaminación ambiental. Políticas ambientales. Plomo. Test de inteligencia. Economía ambiental. Evaluación de la discapacidad. Evaluación del Impacto en la Salud.

Correspondencia

José María Ordóñez Iriarte

C/Ronda de Segovia 52, $1^{\text {a }}$ planta

28005-Madrid. España

josemaria.ordonez@salud.madrid.org

DOI:

\section{ABSTRACT}

\section{Economic Appraisal of the Impact} of the Ban on the Use of Lead in Gasoline on the Intellectual Quotient of 7-8 Years Old in the Community of Madrid

Background: The repercussions on health of public policies are hard to assess from an economic point of view, which is why this is rarely done. The purpose of this study was to financially quantify the benefits of reducing blood lead levels in children aged 7-8 years in the Community of Madrid (Spain) as a result of the ban on the use of lead in gasoline.

Methods: The decrease the intellectual quotient (IQ) points was calculated through two studies on children aged 7-8 years according to their blood lead levels. A geometric mean of $3.8 \mu \mathrm{g} / \mathrm{dl}$ was obtained in the $1995 \mathrm{study}$ and of $0.9 \mu \mathrm{g} / \mathrm{dl}$ in the 2010 study. The net increase in IQ was measured in terms of productivity gained throughout the working life as per the methods of Schwartz and Salkever.

Results: The decrease in blood lead levels in these children prevented a loss of between 135,391 and 144,153 IQ points; the current economic valuation of these points in terms of the productivity gained throughout the working life of this cohort of children was estimated to fall within the $€ 626.4 \mathrm{~m}-€ 865.4 \mathrm{~m}$ range (2009).

Conclusions: The figures of the economic benefits derived from the decision to ban leaded gasoline are very high.

Keywords: Childood. Environmental pollution. Gasoline. Lead. Public policy. Environmental economics. Disability evaluation. Health Impact assessment. 


\section{INTRODUCCIÓN}

Aunque el plomo es un metal usado por el hombre desde antiguo, las grandes emisiones al medio ambiente se produjeron en la segunda mitad del siglo XX, siendo la principal fuente el plomo contenido en las gasolinas ${ }^{1}$.

Los problemas de contaminación atmosférica vinculados al tráfico de vehículos comenzaron en la década de los años 1960, lo que se tradujo en Estados Unidos (EEUU) en la publicación en el año 1963 del Clean Air $A c t .^{2}$ En Europa en los años 70 se comenzaron a adoptar medidas de protección de la calidad del aire, sobre todo del plomo utilizado como antidetonante en las gasolinas, señalado como una amenaza, fundamentalmente para los niños ${ }^{3}$.

La Unión Europea cuantificó la reducción de plomo en la sangre de los niños europeos para el periodo 1978-1988 en un rango que se encontraba entre el 25 y el $45 \%$. A ese descenso también contribuyó que durante el mismo periodo de tiempo se sustituyó el plomo para las soldaduras de las latas de conservas por otros métodos ${ }^{3}$.

En 1998, los ministros de Sanidad de los países europeos fijaron el compromiso de eliminar el plomo de las gasolinas en $2005^{4}$. La Unión Europea adelantó esa fecha al año 2000 y España solicitó una moratoria hasta el 1 de agosto del año 2001, día en el que entró en vigor la nueva normativa ${ }^{5}$. Esta medida se tradujo en una reducción de más de 300.000 $\mathrm{kg}$ /año de emisión de plomo a la atmósfera en la Comunidad de Madrid ${ }^{5}$. Llop et $\mathrm{al}^{6}$, en 2013, observaron una reducción en los niveles de plomo en el aire de diversas ciudades.

Los efectos neurológicos constituyen una de las afectaciones más graves sobre la salud provocadas por el plomo. Se manifiestan en retraso cognitivo e intelectual y en alteraciones de la conducta, -niños hiperactivos y niños con carácter violento- ${ }^{7-9}$. Needelman, en1979, mostró, tras controlar por 39 covariables, que los niños con altos niveles de plo- mo en diente $(>24 \mathrm{ppm})$ en relación a los que presentaban menos $(<6 \mathrm{ppm})$, tenían 6 puntos menos de cociente intelectual $(\mathrm{CI})^{10}$.

Existe abundante literatura que relaciona los niveles de plomo en sangre con pérdida de capacidad mental, medida en reducción del CI, confirmando que el plomo es capaz de dañar permanentemente el sistema nervioso central, incluso a niveles de 1 a $3 \mu \mathrm{g} / \mathrm{dL}$, para los que no se han evidenciado síntomas ${ }^{11-16}$. Los metanálisis realizados con estudios de cohortes y de seguimiento sugieren que el CI se reduce entre 1 y 5 puntos por cada $10 \mu \mathrm{g} /$ $\mathrm{dL}$ de incremento de plomo en la sangre de los niños ${ }^{1,17}$.

Actualmente se considera que no hay ningún nivel de plomo en sangre que sea seguro en los niños ${ }^{18}$. Por ello, los Centers for Disease Control and Prevention (CDC) $)^{19}$ redujeron a $5 \mu \mathrm{g} / \mathrm{dL}$ el nivel de referencia en la infancia (que es el percentil 97,5 de la distribución de plomo en sangre de los niños de 1 a 5 años de EEUU), siguiendo los informes elaborados por el Advisory Committee on Childhood Lead Poisoning Prevention (ACCLPP, 2012 $)^{20}$.

La afectación del plomo en el CI de los niños puede producir importantes efectos económicos a largo plazo, en términos de su capacidad de producción futura, y la estimación de su valor monetario puede ser muy relevante para orientar las prioridades en materia de salud ambiental. Una de las primeras aproximaciones al cálculo de los beneficios sociales derivados de la reducción de los niveles de plomo en la sangre de los niños fue el modelo desarrollado por Schwartz ${ }^{21}$, revisado por Salkever en $1995^{22}$. A partir de los modelos anteriores en 2009 Gould $^{23}$ llevó a cabo un análisis de los beneficios económicos y sociales del control de los riesgos derivados del plomo en EEUU y Pichery en $2011^{24}$, por primera vez en Europa y siguiendo a su vez el esquema de Gould, calculó los beneficios derivados de la reducción de los niveles de plomo en la sangre de los niños franceses. 
Para estas estimaciones Schwartz ${ }^{21}$ recogió las distintas relaciones que se establecen entre plomo y las variables que se ven afectadas por él y calculó las posibles pérdidas salariales por el incremento del plomo en sangre que tendría un niño a lo largo de su vida laboral. Efectos directos (CI, salarios) e indirectos (escolarización, participación en el mercado laboral).

Estimó que la pérdida de 1 punto de CI se asocia a una reducción de un 1,76\% de los ingresos salariales a lo largo de toda la vida; un $0,5 \%$ lo sería de forma directa y el resto, el $1,26 \%$, de forma indirecta. De ese 1,26 $\%$, un $0,79 \%$ por efecto de la disminución de aprendizaje durante la escolarización y un $0,47 \%$ por efecto de la menor productividad en el mercado laboral.

Según Salkever, la pérdida de 1 punto del CI se asocia con una reducción de 1,93\% de los ingresos salariales a lo largo de toda la vida en los varones y de $3,23 \%$ en las mujeres ${ }^{22}$. El efecto directo disminuye ganancias de un $1,24 \%$ en los varones y de un $1,40 \%$ en las mujeres. Los efectos indirectos son estimados en $0,49 \%$ y $1,10 \%$, en relación al aprendizaje y en un 0,20 y un $0,73 \%$, respectivamente, en relación a la productividad. Con una media ponderada según la contribución por sexo al mercado laboral (65\% hombres y $35 \%$ mujeres) se produce una reducción de ingresos medios de 2,37\%, estimación ligeramente mayor que la que hace Schwartz.

En los estudios realizados en la Comunidad de Madrid se evidencia un descenso en los valores del plomo en sangre en los niños de 7 a 8 años que pasaron desde los $3,8 \mu \mathrm{g} /$ dL de media geométrica en $1995^{25}$ hasta los $0,9 \mu \mathrm{g} / \mathrm{dL}(1,1 \mu \mathrm{g} / \mathrm{dL}$ de media aritmética $)$ en $2010^{26}$. Sin embargo en España no se han realizado estudios económicos de los beneficios de estas políticas de salud pública medioambiental.

Por ese motivo el objetivo de este trabajo es hacer una valoración monetaria del impacto producido por los puntos de cociente inte- lectual cuya pérdida se ha evitado, según los niveles de plomo en sangre de los niños de la Comunidad de Madrid en 1995 y en 2010, derivados fundamentalmente de la aplicación de la política de prohibición del plomo de las gasolinas.

\section{MATERIAL Y MÉTODOS}

En este estudio, realizado desde la perspectiva comunitaria, se han tenido en consideración los siguientes aspectos:

Niveles de plomo en sangre. Para la realización de este trabajo se partió de dos estudios llevados a cabo en $1995^{25}$ y $2010^{26}$. La población de niños de 7 y 8 años en la Comunidad de Madrid era de 103.378 en $1995^{27}$ y presentaba una media geométrica de plomo en sangre de 3,8 $\mu \mathrm{g} / \mathrm{dL}$ (DE: 0,2); en 2010, los niños de esa edad eran $128.480^{28}$, con una media aritmética de plomo en sangre de $1,1 \mu \mathrm{g} /$ dL(DE: 0,7$)$ y geométrica de $0,9 \mu \mathrm{g} / \mathrm{dL}(\mathrm{DE}$ : $1,1)^{26}$.

Valoración de la reducción en el cociente intelectual. Se utilizó la metodología definida por Lanphear ${ }^{13}$ que ajustó un modelo de relación entre plomo en sangre de los niños y CI, controlando por el conjunto de covariables que podían confundirla. La relación dosisrespuesta se ajustó a un modelo loglinear en el que los mayores efectos del plomo sobre el CI se produce a niveles más bajos.

En base a estas relaciones se calcularon los puntos de CI que se perdieron en cada uno de los tramos definidos por Lanphear y en cada una de las dos fases del estudio.

Se infirieron los resultados obtenidos en cada una de las muestras estudiadas en cada fase a la población de niños existentes tanto en el año 1995 como en el año 2010, estableciendo que el valor verdadero en la población estaría dentro del 95\% del intervalo definido por valor muestral. Así se sabe qué número de niños hubo en cada uno de los rangos de concentración de plomo en sangre definidos por Lanphear. 
Análisis económico. Se llevó a cabo una aproximación al cálculo de los beneficios sociales derivados de la reducción de los niveles de plomo en la sangre de los niños en términos del decremento de puntos de CI en los niños de la Comunidad de Madrid, siguiendo los modelos de Schwartz ${ }^{21}$ y Salkever ${ }^{22}$ resumido por Pichery ${ }^{24}$.

En el modelo, los beneficios monetarios se valoraron en términos de costes evitados y de las ganancias en la capacidad de producción, que se reflejan en la siguiente fórmula ${ }^{24}$.

$\mathrm{B}=\mathrm{B}_{\text {médicos }}+\mathrm{B}_{\text {ganancias }}+\mathrm{B}_{\text {educación especial }}+\mathrm{B}_{\text {delincuencia }}+\mathrm{B}_{\text {otros }}$ donde:

B: beneficio total

$\mathrm{B}_{\text {médicos }}$ : costes médicos directos evitados

$\mathrm{B}_{\text {ganancias }}$ : capacidad de producción ganada

$\mathrm{B}_{\text {educación especiall }}$ costes de educación especial evitados

$\mathrm{B}_{\text {delincuencia: }}$ : costes derivados de la delincuencia juvenil evitados

$\mathrm{B}_{\text {otros }}:$ costes intangibles evitados

El valor de la corriente de ingresos salariales a lo largo de la vida de un individuo en España, para el año 2009 era de 315.973 euros para los hombres y 202.149 euros para las mujeres, teniendo en cuenta los siguientes supuestos ${ }^{29-33}$ :

- La vida laboralmente activa de una persona en la Comunidad de Madrid va desde los 16 a los 66 años, según la normativa de jubilación reciente ${ }^{29}$.

- Las tasas de ocupación se tomaron de las Tablas del Instituto Nacional de Estadística (INE), diferentes para varones y mujeres ${ }^{30}$.

- El salario medio de un varón en el año 2009 en España fue de 13.682 euros.

- El flujo de rentas de una persona nacida hoy en España bajo los supuestos anteriores será de 1.181.193 euros (varones) y de 935.489 euros (mujeres), sin ajustar por tasa de empleo, y de 701.861 euros (varones) y 438.042 euros (mujeres) tras ajustar por las tasas de empleo.

- Los valores futuros se descontaron a una tasa de descuento del $3 \%{ }^{31,32}$.

- Consideramos como caso base la aplicación de un crecimiento anual de la productividad del $1 \%{ }^{33}$.

\section{RESULTADOS}

En el intervalo entre 2,4 y $10 \mu \mathrm{g} / \mathrm{dL}$ se perderían de media 3,9 (IC $95 \%, 2,4-5,3$ ) puntos de CI si todos los niños tuviesen de valor medio $10 \mu \mathrm{g} / \mathrm{dL}$ de plomo en sangre. Como en ese intervalo tenían de media 4,2 $\mu \mathrm{g} / \mathrm{dL}$ en 1995 y $2,6 \mu \mathrm{g} / \mathrm{dL}$ en 2010 , la pérdida media de puntos de CI por niño fue de 1,53 y 0,22 respectivamente. En el rango de 10,1 a $20 \mu \mathrm{g} / \mathrm{dL}$ se pierden 5,8 puntos: a los 3,9 puntos de CI, por tener ya $10 \mu \mathrm{g}$ / dL de plomo, habría que sumarle 1,9 (IC 95 $\%, 1,2-2,6)$, si la media de plomo en sangre fuese de $20 \mu \mathrm{g} / \mathrm{dL}$. En el caso del estudio de 1995 supuso una pérdida de puntos de CI de 4,28. En el estudio de 2010 no hubo niños con niveles de plomo en sangre por encima de los $10 \mu \mathrm{g} / \mathrm{dL}$. Por último, para el rango entre 20,1 y $30 \mu \mathrm{g} / \mathrm{dL}$, la pérdida es de 6,9 puntos: a los 5,8 anteriores se añadía 1,1 (IC $95 \%, 0,7-1,5)$. Se desconoce el impacto que el plomo tiene en el CI por debajo de los 2,4 $\mu \mathrm{g} / \mathrm{dL}$ (tabla 1).

En la tabla 2 se presentan el número de niños de 7-8 años por cada tramo de concentración de plomo y su porcentaje. Conjugando los datos de las tablas 1 y 2 , hallamos que el número de puntos totales de CI perdidos se encontró entre los 135.672 y los 147.188 , en 1995 y entre 281 y 3.035 en 2010 (tabla 3 ).

Por lo tanto, el balance de puntos de CI que se evitó perder por la reducción de los niveles de plomo en la sangre de los niños para la cohorte de 7-8 años, en la Comunidad de Madrid en el año 2010 se estimó entre 135.391 y 144.153 puntos (tabla 4 ). 


\begin{tabular}{|c|c|c|c|c|c|}
\hline \multicolumn{6}{|c|}{ 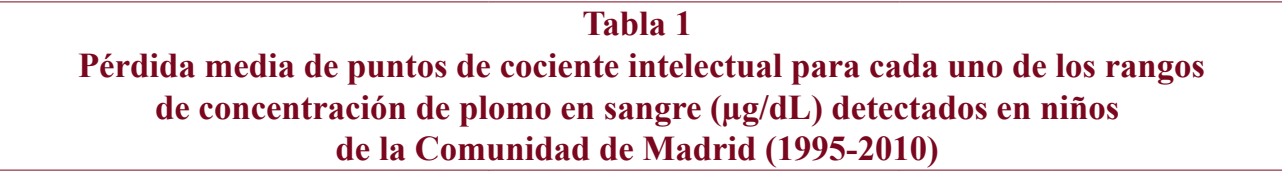 } \\
\hline \multicolumn{2}{|c|}{ Lanphear et al 1995} & \multicolumn{2}{|c|}{ Estudio año 1995} & \multicolumn{2}{|c|}{ Estudio año 2010} \\
\hline $\begin{array}{c}\text { Tramos de } \\
\text { concentración } \\
\text { de } \mathrm{Pb} \text { en sangre } \\
(\mu \mathrm{g} / \mathrm{dL})\end{array}$ & $\begin{array}{c}\text { Pérdida media } \\
\text { de puntos } \\
\text { del cociente intelectual } \\
\text { por tramo de plomo } \\
\text { en sangre }\end{array}$ & $\begin{array}{l}\text { Media de } \\
\text { plomo } \\
\text { en sangre } \\
(\mu \mathrm{g} / \mathrm{dL})\end{array}$ & $\begin{array}{c}\text { Pérdida media } \\
\text { de puntos del } \\
\text { cociente intelectual } \\
\text { por niño }\end{array}$ & $\begin{array}{l}\text { Media } \\
\text { de plomo } \\
\text { en sangre } \\
(\mu \mathrm{g} / \mathrm{dL})\end{array}$ & $\begin{array}{c}\text { Pérdida media } \\
\text { de puntos del } \\
\text { cociente intelectual } \\
\text { por niño }\end{array}$ \\
\hline$<2,4$ & no determinable & 1,7 & - & 1,0 & - \\
\hline $2,4-10$ & & 4,2 & 1,53 & 2,6 & \\
\hline $10,1-20$ & $3,9+(1,9)=5,8$ & 11,5 & $3,9+0,38=4,28$ & - & - \\
\hline$>20$ & $3,9+1,9+(1,1)=6,9$ & & - & & \\
\hline Total & & 3,8 & & 1,1 & - \\
\hline
\end{tabular}

Tabla 2

Porcentaje y número de niños de 7 y 8 años de la Comunidad de Madrid según rangos de concentración de plomo en sangre $(\mu \mathrm{g} / \mathrm{dL})$ (Inferencia poblacional).

Años 1995 y 2010

\begin{tabular}{|c|c|c|c|c|c|c|c|c|}
\hline \multirow{3}{*}{$\begin{array}{l}\text { Tramos de } \\
\text { concentración } \\
\text { de plomo } \\
\text { en sangre } \\
(\mu \mathrm{g} / \mathrm{dL})\end{array}$} & \multicolumn{4}{|c|}{ Estudio año 1995} & \multicolumn{4}{|c|}{ Estudio año 2010} \\
\hline & \multirow{2}{*}{$\begin{array}{c}\% \text { de niños } \\
\text { en cada tramo de } \\
\text { concentración } \\
\text { de plomo } \\
\text { en sangre }\end{array}$} & \multicolumn{3}{|c|}{$\begin{array}{l}\text { Número de niños estimados } \\
\text { en cada tramo de concentración } \\
\text { de plomo en sangre }\end{array}$} & \multirow{2}{*}{$\begin{array}{l}\text { \% de niños en } \\
\text { cada tramo de } \\
\text { concentración } \\
\text { de plomo en } \\
\text { sangre }\end{array}$} & \multicolumn{3}{|c|}{$\begin{array}{l}\text { Número de niños estimados } \\
\text { en cada tramo de concentración de } \\
\text { plomo en sangre }\end{array}$} \\
\hline & & $\begin{array}{l}\text { Límite } \\
\text { inferior }\end{array}$ & Medio & $\begin{array}{l}\text { Límite } \\
\text { superior }\end{array}$ & & $\begin{array}{l}\text { Límite } \\
\text { inferior }\end{array}$ & Medio & $\begin{array}{l}\text { Límite } \\
\text { superior }\end{array}$ \\
\hline$<2,4$ & $11,3( \pm 2,7)$ & 8.890 & 11.682 & 14.473 & $94,1( \pm 2,6)$ & 117.559 & 120.900 & 124.240 \\
\hline 2,4-10 & $88,3( \pm 2,8)$ & 88.387 & 91.282 & 94.176 & $5,9( \pm 4,9)$ & 1.284 & 7.580 & 13.876 \\
\hline $10,1-20$ & $0,4( \pm 0,3)$ & 103 & 414 & 724 & 0 & 0 & 0 & 0 \\
\hline$>20$ & 0 & 0 & 0 & 0 & 0 & 0 & 0 & 0 \\
\hline Total & & & 103.378 & & & & 128.480 & \\
\hline
\end{tabular}

\begin{tabular}{|c|c|c|c|c|c|c|}
\hline \multicolumn{7}{|c|}{$\begin{array}{c}\text { Tabla } 3 \\
\text { Pérdida de puntos de CI en los niños de } 7 \text { y } 8 \text { años de la Comunidad de Madrid } \\
\text { en } 1995 \text { y } 2010 \text { según rangos de concentración de plomo en sangre }(\mu \mathrm{g} / \mathrm{dL})\end{array}$} \\
\hline \multirow{3}{*}{$\begin{array}{c}\text { Tramos de concentración } \\
\text { de plomo en sangre } \\
(\mu \mathrm{g} / \mathrm{dL})\end{array}$} & \multicolumn{3}{|c|}{ Estudio año 1995} & \multicolumn{3}{|c|}{ Estudio año 2010} \\
\hline & \multicolumn{3}{|c|}{$\begin{array}{l}\text { Puntos de CI perdidos según tramos } \\
\text { de concentración de plomo en sangre }\end{array}$} & \multicolumn{3}{|c|}{$\begin{array}{l}\text { Puntos de CI perdidos según tramos de } \\
\text { concentración de plomo en sangre }\end{array}$} \\
\hline & Límite inferior & Medio & $\begin{array}{c}\text { Límite } \\
\text { superior }\end{array}$ & $\begin{array}{l}\text { Límite } \\
\text { inferior }\end{array}$ & Medio & Límite superior \\
\hline$<2,4$ & - & - & - & - & - & - \\
\hline $2,4-10$ & 135.232 & 139.661 & 144.089 & 281 & 1.658 & 3.035 \\
\hline $10,1-20$ & 440 & 1.772 & 3.099 & - & - & - \\
\hline$>20$ & - & - & - & - & - & - \\
\hline Total & 135.672 & 141.433 & 147.188 & 281 & 1.658 & 3.035 \\
\hline
\end{tabular}


Esta ganancia (o no pérdida) es media pero no se distribuye de igual forma entre todos los niños. Los más afectados serían aquellos que ya estuviesen en una situación cercana al límite intelectual.

Capacidad de producción ganada a lo largo del tiempo. Según la valoración económica utilizada, la pérdida de 1 punto de CI en España fue de 5.561 euros en los varones (315.973 euros $\times 1,76 \%$,) y de 3.558 euros en las mujeres $(202.149 \times 1,76 \%)$, con una media de 4.560

\begin{tabular}{|c|c|c|c|}
\hline \multicolumn{4}{|c|}{$\begin{array}{l}\text { Tabla } 4 \\
\text { Balance de pérdida de puntos de cociente } \\
\text { intelectual evitados en los niños de } 7 \text { y } 8 \\
\text { años de la Comunidad de Madrid para el } \\
\text { periodo 1995-2010 }\end{array}$} \\
\hline \multirow{2}{*}{$\begin{array}{c}\text { Año } \\
\text { del } \\
\text { estudio }\end{array}$} & \multicolumn{3}{|c|}{ Pérdida de puntos de CI } \\
\hline & $\begin{array}{l}\text { Límite } \\
\text { inferior }\end{array}$ & $\begin{array}{l}\text { Límite } \\
\text { medio }\end{array}$ & $\begin{array}{c}\text { Límite } \\
\text { superior }\end{array}$ \\
\hline 1995 & 135.672 & 141.433 & 147.188 \\
\hline 2010 & 281 & 1.658 & 3.035 \\
\hline Balance & 135.391 & 139.775 & 144.153 \\
\hline
\end{tabular}

\section{Tabla 5}

Capacidad de producción ganada de acuerdo a los puntos de CI evitados por la reducción de la concentración de plomo en sangre de los niños de la Comunidad de Madrid: balance 1995-2010

\begin{tabular}{|c|c|c|c|}
\hline \multirow{2}{*}{$\begin{array}{l}\text { Tramos de concentración } \\
\text { de plomo en sangre } \\
(\mu \mathrm{g} / \mathrm{dL})\end{array}$} & \multirow{2}{*}{$\begin{array}{c}\text { Pérdidas evitadas } \\
\text { de puntos de CI en niños } \\
\text { de } 1995 \text { a-2010 } \\
\text { (valor medio) }\end{array}$} & \multicolumn{2}{|c|}{ Capacidad de producción ganada (millones de euros) } \\
\hline & & $\begin{array}{l}\text { Límite inferior } \\
\text { (Schwartz) }\end{array}$ & $\begin{array}{c}\text { Límite superior } \\
\text { (Salkever) }\end{array}$ \\
\hline$<2,4$ & - & - & - \\
\hline $2,4-10,0$ & 138.003 & 629,3 & 855,1 \\
\hline $10,1-20,0$ & 1.772 & 8,1 & 10,3 \\
\hline$>20,0$ & - & - & - \\
\hline Total & 139.775 & 637,4 & 865,4 \\
\hline
\end{tabular}

euros. Con la estimación de Salkever fue de 7.552 euros para varones y de 4.831 euros para las mujeres. La media en este caso fue de 6.192 euros.

Con los datos de las tablas 3 y 4 , que recoge los puntos de CI que se evitaron perder en el periodo 1995 a 2010 por cada tramo, se estimaron los beneficios económicos teniendo en cuenta el valor de cada punto de CI. Estos beneficios se estimaron en un rango entre 637,4 y 865,4 millones de euros (valor de 2009) para toda la vida laboral (tabla 5).

\section{DISCUSIÓN}

La reducción del plomo en la sangre de los niños contribuye a evitar que se pierdan entre 135.391 y 144.153 puntos de CI entre los niños de 7-8 años de la Comunidad de Madrid. $\mathrm{Su}$ valoración en términos económicos ayuda a justificar la implementación de políticas como fue la prohibición del plomo de las gasolinas.

Los niños de la Comunidad de Madrid presentaron en el año 1995 niveles de plomo en sangre similares a los de países y zonas desarrolladas del mismo periodo ${ }^{3,34-38}$. Estos valores se han ido reduciendo, habiéndose encontrado el valor medio de $1,1 \mu \mathrm{g} / \mathrm{dL}$ en niños de 0 a 15 años en el estudio realizado en el año $2010^{26}$. Otros estudios realizados en niños recién nacidos en Madrid y en otras regiones españolas, Asturias, Gipuzkoa, Sabadell y Valencia, llevados a cabo entre el año 2003 y 2008, mostraron similar reducción ${ }^{6,38}$. Si esta reducción de los niveles de plomo en la sangre de los niños es presentada en términos de daño evitado y de beneficios económicos, se puede contribuir a que las políticas de salud pública (o las políticas sectoriales que inciden en ella, como es el caso) pierdan esa parte de invisibilidad social que tienen ${ }^{39}$. 
En el caso de EE UU, la media de puntos de CI perdidos por niño para la cohorte de $1 \mathrm{a}$ 6 años en el año 2006 fue de 1,6 $6^{23}$. en Francia de $0,6^{24}$ en el año 2008 y para el estudio de la Comunidad de Madrid de 0,01 puntos de CI por persona, en el año $2010^{26}$. Estas diferencias se explican porque los niños de EEUU y de Francia parten con una carga de plomo en sangre mayor que los de la Comunidad de Madrid. En estos dos países, a la exposición al plomo de las gasolinas que sufrieron los niños hay que añadirle la exposición al descascarillado de las pinturas de las $\operatorname{casas}^{23,24}$, situación que no ocurrió en España por tener una legislación restrictiva para su uso en el interior de las viviendas desde $1928^{40} \mathrm{y}$ algunas otras fuentes potenciales como el agua, etcétera.

Es reseñable que el coste de 1 punto de CI calculado para España, estuviera ajustado al valor de 2009, entre 4.560 y 6.192 euros, muy alejado de los 17.363 estimados por Gould en $E_{E U U}{ }^{23}$ y utilizado por Pichery et al en Francia ${ }^{24}$.

No se valoraron otros costes sociales derivados de la exposición al plomo, como los de la educación especial o los debidos a la delincuencia pero, según otros autores que sí lo han hecho, su cuantía es pequeña en relación a la capacidad de producción ganada que se calculó en este estudio ${ }^{23,24}$.

En todo caso, la estimación de los costes sociales evitados realizada en este estudio representa su límite inferior, puesto que los niveles de plomo provocan otros efectos adicionales, como enfermedades cardiovasculares o cáncer, que pueden llevar a una muerte prematura y que no se calcularon ${ }^{24}$. Además, habría que incluir los efectos que el plomo provoca por debajo de los $2,4 \mu \mathrm{g} / \mathrm{dL}$ que, hasta la fecha, no han sido cuantificados.

Este estudio no se planteó como una evaluación económica estricta. Se centró exclusivamente en la medición de los beneficios de una política, sin compararlos con otros costes como los de adaptación de los procesos de producción, etcétera, dado el periodo de tiempo tan largo desde su implantación.

Existen antecedentes de evaluación económica de programas de salud pública que justificarían precisamente su implementación porque son rentables socialmente en términos de coste-beneficio o coste-efectividad, es decir, eficientes ${ }^{41,42}$. Sin embargo, la evaluación económica no es una técnica que se utilice con frecuencia en el ámbito de la salud pública y de la sanidad ambiental ${ }^{43}$. A ello no es ajeno el escaso desarrollo en nuestro país de medidas de efectividad como los Años de Vida Ajustados por Calidad (AVACs) o Quality-adjusted Life Years (QALYs) en su acrónimo inglés más conocido, y también por la ausencia de bases de datos de costes unitarios. Además, los beneficios de las políticas públicas tienen un componente importante de externalidad, es decir, afectan a muchas personas sin mediar el mecanismo del mercado, lo que hace muy difícil su medida y valoración adecuadas $^{44}$. Además, hay problemas metodológicos específicos en la evaluación económica de las políticas de salud pública debidos a la complejidad de la salud pública en sí mismas: la nada fácil cuestión de la atribución de los efectos a las políticas, la dificultad de realizar ensayos aleatorios controlados así como el carácter intersectorial de muchas políticas, que requieren una consideración más amplia de los costes y beneficios que recaen sobre otros sectores diferentes al sanitario ${ }^{45,46}$. Esta ausencia podría explicar la escasa importancia que reciben las políticas de salud pública en relación con las políticas asistenciales, que generalmente cuentan con evaluaciones de eficacia y, en muchos casos, con evaluaciones económicas de coste efectividad.

Quizá el efecto más relevante para los individuos afectados por el plomo y sus familiares sea el coste intangible de la reducción en el cociente intelectual. Pichery et $\mathrm{al}^{24}$ se refieren a él como pretium doloris. La medida de este efecto podría venir recogida en los cambios en la calidad de vida asociada a la salud a lo largo de la vida, normalmente medidos en 
QALYs, pero incluso los QALYs pueden no recoger totalmente esta idea si se contempla la pérdida de bienestar en sentido amplio.

El estudio presentó limitaciones. Entre otras, las derivadas del uso de los valores de plomo de dos estudios transversales en los que la selección de los participantes en las muestras poblacionales fue diferente: la de 1995 en el ámbito escolar y la de 2010 en el hospitalario, lo que le resta representatividad. Incluso, en este último caso, se podría pensar que al ser niños que van a consulta podrían tener mayor probabilidad de presentar una enfermedad asociada al plomo. No obstante, de existir este sesgo, provocó una medición de la plumbemia media mayor de la que realmente tenían los niños del estudio de 2010, por lo cual estaría infraestimado el ahorro de puntos de CI y su valoración económica. Tampoco se consideró que tuviera repercusión el que la muestra de niños del estudio del año 2010 llegó hasta los 15 años de edad, ya que las medias globales de edad de ambos grupos eran similares y no existían diferencias estadísticamente significativas entre ellos.

Vista la relevancia de los beneficios económicos derivados de la capacidad de producción ganada al evitar perder puntos de CI, que se estimó entre 637,4 y 865,4 millones de euros para esta cohorte de niños de la Comunidad de Madrid, se constata que analizar los beneficios sanitarios en términos monetarios aumenta la visibilidad del impacto de las políticas de salud pública, lo que puede contribuir a orientar la toma de decisiones en pie de igualdad con otros ámbitos de la sanidad.

\section{BIBLIOGRAFÍA}

1. Agency for Toxic Substances and Disease Registry. Toxicological Profile for Lead (Update). US Dept of Health and Human Services, Atlanta, 2007 [Citado el 10/11/2014]. Disponible en http://www.atsdr.cdc.gov/ toxprofiles/tp13.pdf.

2. United States Code. Clean Air Act, 1970 [Citado el 10/11/2014]. Disponible en http://www.epw.senate.gov/ envlaws/cleanair.pdf.
3. World Health Organization. Levels of Lead in Children's blood. European Environmental Health Information System (ENHIS) [Citado el 10/11/2014]. Disponible en http:/www.euro.who.int/_data/assets/pdf_file/0003/97050/4.5.-Levels-of-lead-in-childrens-blood-EDITING_layouted.pdf?ua $=1$.

4. Diario Oficial de la Unión Europea. Directiva 98/70/CE del Parlamento Europeo y del Consejo, de 13 de octubre de 1998, relativa a la calidad de la gasolina y el gasóleo y por la que se modifica la Directiva 93/12/CEE del Consejo. DOCE núm L 350 de 28/12/1998

5. Boletín Oficial del Estado. Real Decreto 785/2001, de 6 de Julio, por el que se adelanta la prohibición de comercialización de las gasolinas con plomo y se establecen las especificaciones de las gasolinas que sustituirán a aquellas. BOE núm 162 de 7/7/2001.

6. Llop S, Porta M, Martínez MD, Aguinagalde X, Fernández MF, Fernández-Somoano A. et al. Estudio de la evolución de la exposición a plomo en la población infantil española en los últimos 20 años. ¿Un ejemplo no reconocido de "salud en todas las políticas"? Gac Sanit. 2013; 27(2):140-55.

7. Bellinger DC. Lead. Pediatrics. 2004;113:16-22.

8. Shober SE, Mirel LB, Graubard BI, Brody DJ, Flegal KM. Blood lead levels and death from all causes, cardiovascular disease, and cancer: results from the NHANES III mortality study. Environ Health Perspect. 2006; 114(10):1538-41.

9. Yuan W, Holland SK, Cecil KM, Dietrich KN, Wessel SD, Altaye $M$ et al. The impact of early childhood lead exposure on brain organization: a functional magnetic resonance imaging study on language function. Pediatrics. 2006; 118(3):971-77.

10. Needelman HL Gunnoe C, Leviton A, Reed R, Peresie H, Maher C et al. Deficits in psychologic and classroom performance of children with elevated dentine lead levels. N Engl J Med. 1979; 300(13):689-95.

11. Lidsky T, Schneider JS. Lead neurotoxicity in children: basis mechanisms and clinical correlates. Brain. 2003;126(13):5-19.

12. Schwartz J. Low-level lead exposure and children's IQ: A meta-analysis and search for a threshold. Environ Res. 1994;65(1):42-55.

13. Lanphear BP, Hornung R, Khoury J, Yolton K, Baghurst P, Bellinger D. Low-Level environmental lead exposure and children's intellectual function: an international pooled analysis. Environ Health Perspect. $2005 ; 113(7): 894-9$. 
14. Canfield RL, Henderson CR, Cory-Slechta DA, Cox C, Jusko TA, Lanphear BP. Intellectual impairment in children with blood lead concentrations below $10 \mu \mathrm{g}$ per deciliter. N Engl J Med. 2003;348(16):1517-26.

15. Bellinger D, Leviton A, Allred E, Rabinowitz M. Preand postnatal lead exposure and behavior problems in school-aged children. Environ Research. 1994;66(1):1230 .

16. Bellinger D, Leviton A, Waternaux C. Lead, IQ and social class. Int J Epidemiol. 1989; 18(1):180-5.

17. McMichael AJ, Baghurst PA, Vimpani GV, Wigg NR, Robertson EF, Tong Sl. Tooth lead levels and IQ in school-age children: The Port Pirie cohort study. Am J Epidemiol. 1994; 140(6):489-99.

18. American Academy of Pediatrics Committee on Environmental Health. Lead Exposure in children: prevention, detection, and management. Pediatrics. 2005;116(4):103646 .

19. Center for Diseases Control and Prevention. CDC Response to Advisory Committee on Childhood Lead Poisoning Prevention Recommendations in "Low Level Lead Exposure Harms Children: A Renewed Call of Primary Prevention" Centers for Disease Control and Prevention [Citado el 10/11/2014]. Disponible en http://www.cdc. $\mathrm{gov} / \mathrm{nceh} / \mathrm{lead} / \mathrm{acclpp} / \mathrm{cdc}$ response_lead_exposure_recs. pdf.

20. Advisory Committee on Childhood Lead Poisonong Prevention. Low Level Lead Exposure Harms Children: A Renewed Call for Primary Prevention Report of the Advisory Committee on Childhood Lead Poisoning Prevention of the Centers for Disease Control and Prevention [Citado el 10/11/2014]. Disponible en http://www.cdc.gov/nceh/ lead/ACCLPP/Final_Document_030712.pdf.

21. Schwartz J. Societal benefits of reducing lead exposure. Environ Res. 1994;66(1):105-24.

22. Salkever DS. Updated estimates of earnings benefits from reduced exposure of children to environmental lead. Environ Res. 1995; 70(1):1-6.

23. Gould E. Childhood lead poisoning: Conservative estimates of the social and economic benefits of lead hazard control. Environ Health Perspect. 2009;117:1162-7.

24. Pichery C, Bellanger M, Zmirou-Navier D, Glorennec P, Hartemann P, Grandjean P. Childhood lead exposure in France: benefit estimation and partial cost-benefit analysis of lead hazard control. Environ Health. 2011;10(44):1-12.

25. Ordóñez-Iriarte JM, Aparicio-Madre MI, AránguezRuiz E, Rivera Cuadrado, A. Factores determinantes de plomo en sangre en niños de 7-8 años de la Comunidad de Madrid. Acta Pediatr Esp. 2009;67(4):173-81.
26. Ordóñez-Iriarte JM, González-Estecha M, GuillénPérez JJ, Martínez-García MJ, Fernández-Montes BG, Aparicio-Madre MI et al. Factores de riesgo asociados a los niveles de plomo en sangre de niños de la Comunidad de Madrid en 2010. Rev Salud Ambient. 2013; 13(2):167-77.

27. Censo de Población y Viviendas 1991. Instituto Nacional de Estadística. Disponible en http://www.ine.es.

28. Estadísticas del Padrón continuo 2010. Instituto Nacional de Estadística. Disponible en http://www.ine.es.

29. Boletín Oficial del Estado. Ley 27/2011, de 1 de agosto, sobre actualización, adecuación y modernización del sistema de Seguridad Social. BOE num 184 de $2 / 8 / 2011$

30. Encuesta de población activa. Instituto Nacional de Estadística. Disponible en http://www.ine.es.

31. Gold M, Siegel J. Russell L, Weinstein M, editors. Cost-Effectiveness in Health and Medicine. Oxford: Oxford University Press; 1996.

32. López-Bastida J, Oliva J, Antoñanzas F, García-Altés A, Gisbert R, Mar J et al. Propuesta de guía para la evaluación económica aplicada a las tecnologías sanitarias. Gac Sanit. 2010;24(2):154-70.

33. Drummond MF editor. Métodos para la evaluación económica de los programas de asistencia sanitaria. Madrid: Díaz de Santos; 2001.

34. Strömberg U, Schütz A, Skerfving S. Substantial decrease of blood lead in Swedish children, 1978-94, associated with petrol lead. Occup Environ Med. 1995; 52(11):764-69.

35. Centers for Disease Control and Prevention. Update; blood lead levels-United States, 1991-1994. Morb Mortal Wkly Rep. 1997;46(7):141-6.

36. Schwemberger JG, Mosby JE, Doa MJ, Jacobs DE, Ashley PJ, Brody DJ et al. Blood lead levelsUnited States, 1999-2002. Morb Mortal Wkly Rep. 2005;54(20):513-6.

37. González-Estecha M. Martínez-Novillo M, Romero M, Jorge JJ, Gancedo M, Domínguez M. Relación del plomo en sangre con el cociente intelectual y nivel de maduración escolar. En Fundación Mapfre ¿Es el plomo un problema de salud pública en España?. Editorial Mapfre SA. Madrid,1998.

38. Llop S, Aguinagalde X, Vioque J, Ibarluzea J, Guxens M, Casas M, et al. Prenatal exposure to lead in Spain: Cord blood levels and associated factors. Sci Total Environ. 2011; 409(11):2298-305. 
39. Segura A, Martín A. Prevención en la práctica clínica desde la perspectiva de la salud pública y la atención primaria de salud. En: del Llano J, Ortún V, Martín JM, et al editores. Gestión sanitaria. Innovaciones y desafíos. Barcelona. Masson: 1998. páginas 575-91.

40. Gazeta de Madrid. Decreto de 19 de febrero de 1926 , por el que se prohíbe el empleo de cerusa, sulfato de plomo y otros productos que contengan estos pigmentos,para pintar el interior de los edificios. Gazeta de Madrid núm de 12-3-1926.

41. Hutton G. Economic evaluation of Environmental Health interventions to support decision making. Environ Health Insights. 2008; 2:137-55.

42 Hutton G. Considerations in evaluating the cost-effectiveness of environmental health interventions. Sustainable. Development and Healthy Environments Cluster. Geneve: World Health Organization; 2000.

43. Espallargues M, Pons J, Almazán C y de Solá-Morales. La evaluación de tecnologías sanitarias en intervenciones de salud pública: ¿más vale prevenir que curar? Gac Sanit. 2011; 25(Supl 1):40-8.

44. Cabasés JM. La sanidad en España: marco conceptual, análisis de situación y recomendaciones en la perspectiva del 2020. En: Círculo de la Sanidad: Sanidad 2020. Un futuro incierto. Madrid; Círculo de la Sanidad: 2013. p. $45-136$

45. Edwards RT, Charles JM, Lloyd-Williams H. Public Health Economics: A systematic review of guidance for economic evaluation of public health interventions and discussion of key methodological issues. BMC public health. 2013; 13(1):1001-13.

46. Nebot M, López MJ (editores). Evaluación en Salud Pública. Gac Sanit. 2011;25(Supl 1). 